\title{
Dispersion properties of silicon nanophotonic waveguides investigated with Fourier optics
}

\author{
J. Jágerská, ${ }^{1}$ N. Le Thomas, ${ }^{1, *}$ R. Houdré, ${ }^{1}$ J. Bolten, ${ }^{2}$ C. Moormann, ${ }^{2}$ T. Wahlbrink, ${ }^{2}$ J. Čtyroký, ${ }^{3}$ \\ M. Waldow, ${ }^{4}$ and M. Först ${ }^{4}$ \\ ${ }^{1}$ Institut de Photonique et d'Électronique Quantiques, Ecole Polytechnique Fédérale de Lausanne (EPFL), CH-1015 \\ Lausanne, Switzerland \\ ${ }^{2}$ Advanced Microelectronic Center Aachen, AMO GmbH, D-52074 Aachen, Germany \\ ${ }^{3}$ Institute of Photonics and Electronics ASCR, v.v.i., Chaberská 57, 18251 Prague 8, Czech Republic \\ ${ }^{4}$ Institut für Halbleitertechnik, RWTH Aachen University, D-52074 Aachen, Germany \\ *Corresponding author: nicolas.lethomas@epfl.ch \\ Received July 6, 2007; accepted July 19, 2007; \\ posted August 3, 2007 (Doc. ID 84986); published September 11, 2007 \\ We experimentally investigate the dispersion relation of silicon-on-insulator waveguides in the $1.5 \mu \mathrm{m}$ \\ wavelength range by using a technique based on far-field Fourier-space imaging. The phase information of \\ the propagating modes is transferred into the far field either by linear probe gratings positioned $1 \mu \mathrm{m}$ away \\ from the waveguide core or by residual gratings located on the sidewalls of the waveguide. As a result, the \\ dispersion curve of rectangular and slot waveguides as well as the group index dispersion are accurately \\ determined. (C) 2007 Optical Society of America \\ OCIS codes: $070.0070,130.0130,130.2790,130.5990$.
}

By nature, guided modes are isolated from the far field, and therefore the investigation of their physical properties is difficult to carry out with noninvasive techniques. However, residual subnanometer scale imperfections, inherent to the technological processing, always result in optical Rayleigh scattering losses. These optical losses, although as low as $3 \mathrm{~dB} / \mathrm{cm}$ for the state-of-the-art submicrometer silicon wire waveguides whose rms roughness amplitude is $\sim 5 \mathrm{~nm}$ [1], carry information about both the input guided field and the dielectric perturbation inducing the scattering process [2]. An a priori unknown disorder of the scatterers, however, hinders recovery of the optical properties linked to the intrinsic modes of the structure, such as dispersion relation.

Dispersion relation $\omega(k)$, with $\omega$ as the angular frequency and $k$ as the wave vector of the electromagnetic mode, contains comprehensive information about the light propagation in photonic structures, and hence, its knowledge is crucial for control of the light flow. The group velocity deduced from the dispersion relation allows, for instance, identifying the phase-matching conditions between interacting pump and probe or Stokes beams at different wavelengths that must be addressed to increase nonlinear efficiencies [3]. The exploitation of such nonlinear effects in high-index-contrast waveguides is currently an active research area [4,5], and in this context efficient, reliable, and accurate experimental investigation methods are highly appealing. Up to now only interferometric techniques that require specially designed samples and/or cumbersome postprocessing and ultrafast optical setups have been considered to retrieve the experimental dispersive properties of $\mathrm{Si}$ wires [6-9].

In this Letter we experimentally determine the dispersive properties of $\mathrm{Si}$ nanowire and slot waveguides by analyzing the emission diagram either of residual scattering losses or of losses induced by properly engineered probes. We also demonstrate that the current advanced e-beam patterning process still induces coherent optical losses that are associated with periodic subnanometer perturbations of the dielectric map of the waveguides.

Rectangular silicon-on-insulator (SOI) waveguides of 300 and $400 \mathrm{~nm}$ width (labeled as R300 and R400, respectively), as well as two different slot waveguides were fabricated from a $300 \mathrm{~nm}$ thick silicon layer on top of a $1 \mu \mathrm{m}$ thick buried oxide layer. Waveguide patterns were defined by e-beam lithography (EBL) using hydrogen silsesquioxane as a resist and transferred to the silicon layer by reactive-ion etching. The top-view scanning electron microscope (SEM) images of the fabricated structures and their cross-sectional layouts are shown in Fig. 1(a). The lateral profile of the slot waveguides, S70 and S130, consists of two $180 \mathrm{~nm}$ wide silicon sections spaced by an air trench of 70 and $130 \mathrm{~nm}$, respectively. As also shown in Fig. 1(a), linear probe gratings (LPGs) have been etched on both sides of the waveguides at the separation distance of $Y=1 \mu \mathrm{m}$ or $Y=3 \mu \mathrm{m}$ to probe the evanescent tail of the guided field effectively and with minimal disturbance. The $\Lambda=1 \mu \mathrm{m}$ period of the LPGs was carefully chosen to fold the dispersion curve of the

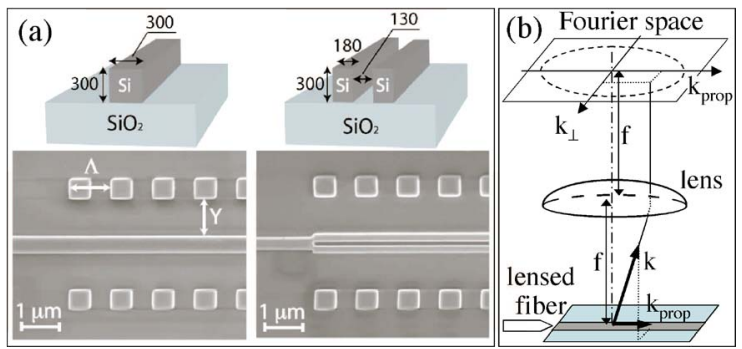

Fig. 1. (Color online) (a) Perspective layout and top-view SEM images of rectangular R300 (left) and slot S130 (right) wire waveguides. The dimensions are given in nanometers. (b) Schematic of the Fourier imaging principle. 
wire waveguides into the light cone, i.e., to couple the guided modes into the radiative continuum [10].

Light from a continuous-wave laser diode tuneable between $1.48-1.66 \mu \mathrm{m}$ is injected via a lensed fiber and an adiabatically tapered structure into the waveguides, as in a conventional endfire setup. The light scattered from the sample surface either at the LPGs or at the waveguide sidewalls is collected by a high numerical aperture (NA) microscope objective $(\mathrm{NA}=0.9)$. For a given excitation wavelength the radiated field is then imaged in the back focal plane of the collecting lens [Fig. 1(b)]. Such an image represents the optical Fourier transform of the field with every single point related to a unique wave vector of the radiated field [11]. The additional knowledge of the reciprocal vector of the LPG together with the conservation law of the parallel wave vector components gives access to the wave vector $k_{\text {prop }}$ of the propagating mode [10]. Recording the $k_{\text {prop }}$ position for each excitation wavelength enables us to reconstruct the entire dispersion curve of the guided modes.

Figures 2(a) and 2(c) show the optical Fourier space diagram of light scattered from the $300 \mathrm{~nm}$ wide rectangular wire waveguide for $Y=1 \mu \mathrm{m}$ and $Y$ $=3 \mu \mathrm{m}$, respectively. For $Y=1 \mu \mathrm{m}$ the pattern exhibits two groups of sharp straight lines $\mathrm{S} 1^{\mathrm{TM}}, \mathrm{S}^{\mathrm{TE}}$, $\mathrm{S} 2^{\mathrm{TM}}$, and $\mathrm{D}^{\mathrm{TM}}, \mathrm{D}^{\mathrm{TE}}$, perpendicular to the onedimensional propagation direction $\vec{k}_{\text {prop }}$ and limited by the pupil of the collecting lens. The vertical profile of the "D" lines exhibits a modulation pattern whereas for the "S" lines the signal is mainly located near the boundary of the pupil of the collecting lens. When $Y=3 \mu \mathrm{m}$, i.e., for a decreased interaction between the guided mode and the LPG, according to the evanescent exponential decay of the mode, the $\mathrm{D}^{\mathrm{TM}}$ and $\mathrm{D}^{\mathrm{TE}}$ lines are missing. These lines are therefore attributed to the radiated fields of the fundamental transverse magnetic (TM) and transverse electric (TE) modes of the wire waveguides scattered by the LPGs. The fields radiated from each LPG interfere in the far field, which produces the modulation observed along the $k_{\perp}$ direction. The TE and TM polarization assignment was performed by polarization analysis in the collecting path.

The far-field S lines, whose corresponding images in the near field are located at the waveguide core, are not related to the scattering at the LPGs. This is evidenced as an increase of the LPG-guide separation has no impact on the intensity profile of these lines as shown in Figs. 2(a) and 2(c), and they are also observed in the absence of the LPG. The $k_{\text {prop }}$ spacing between the $\mathrm{S}_{1}^{\mathrm{TM}}$ and $\mathrm{S}_{2}^{\mathrm{TM}}$ is constant when the wavelength is tuned, and it corresponds to a spatial modulation of $1.28 \mu \mathrm{m}$ that is exactly the dimension of the trapezoidal write subfield pattern used in the EBL process. Therefore, we attribute these lines to the first and second orders of a small corrugation grating on each sidewall of the wire waveguides resulting from the e-beam patterning. For an infinite height of the Si wire, the field radiated from the sidewall would propagate strictly parallel to the sub-

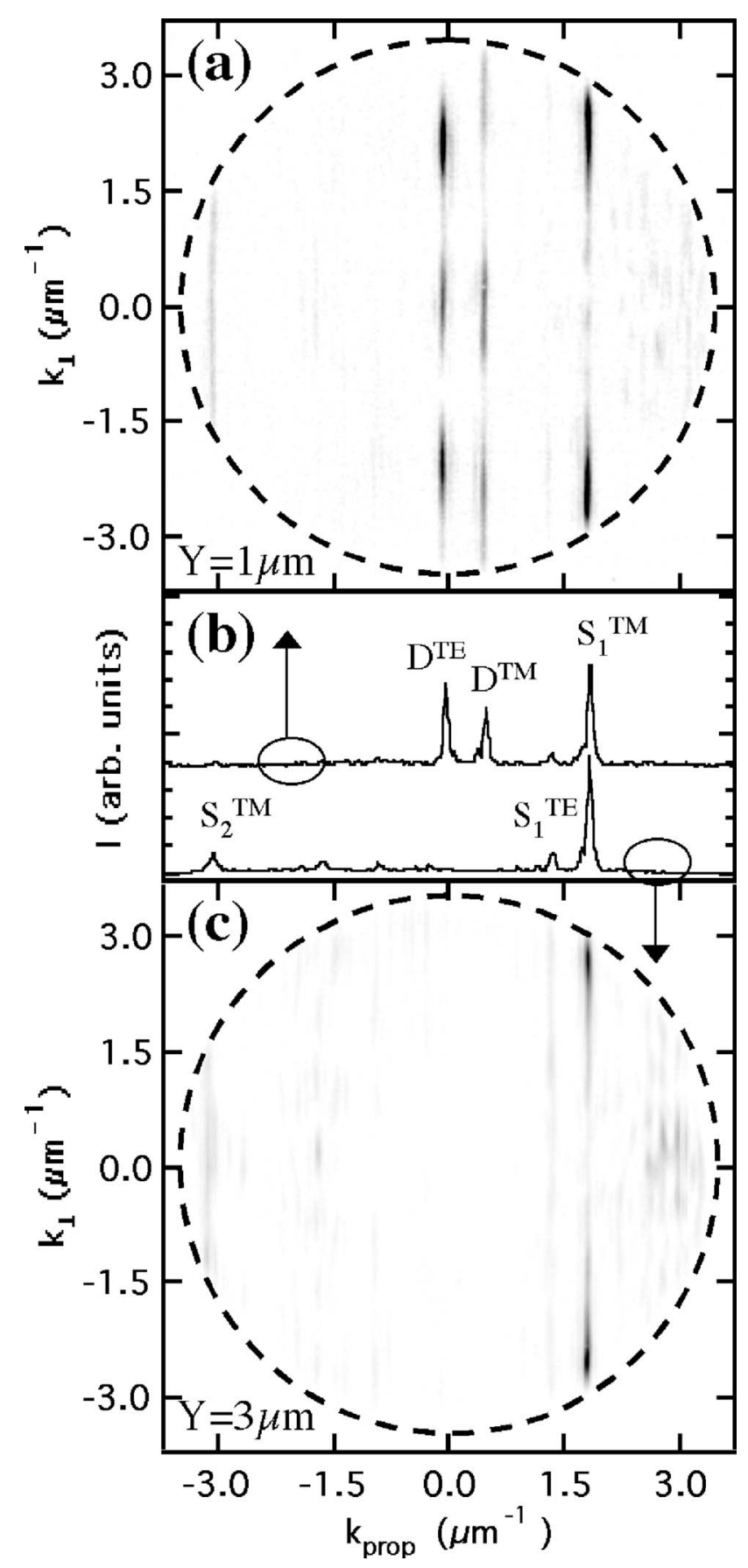

Fig. 2. Optical Fourier space images of the R300 wire waveguide at $\lambda=1620 \mathrm{~nm}$ for (a) $Y=1 \mu \mathrm{m}$ and (c) $Y=3 \mu \mathrm{m}$; (b) corresponding transverse intensity profiles.

strate, i.e., at $90^{\circ}$ from the collection optical axis. As a result no signal would be detected even though the NA of the collecting microscope objective is as high as 0.9 . The strong diffraction resulting from the current $300 \mathrm{~nm}$ height of the Si wire induces, however, an angular spreading of the radiated field whose far-field intensity pattern can be approximate with an Airy function [11]. It follows that some spatial frequencies of the radiated field are detected in the pupil of the 0.9 NA microscope objective. The $k_{\perp}$ profile of the $\mathrm{S}$ lines, limited by the pupil, corresponds to the tail of this Airy far-field pattern whose maximum is located outside the pupil at $k_{\perp}=3.9 \mu \mathrm{m}^{-1}$. 
The residual sidewall grating and the LPG, whose parameters are well defined, can be both considered as first-order perturbative gratings. This implies that the same theoretical model can describe their interaction with the guided mode. Therefore, by using the volume current method [12], the amplitude of the coherent sidewall roughness $d_{s}$ can be estimated from the comparison of the radiative losses induced by the corrugation grating and the LPG. Such an approach provides a rough estimation of $d_{s} \approx 1 \mathrm{~nm}$, which corresponds to a corrugation not detectable by conventional SEM analysis.

From the knowledge of the reciprocal vector of the gratings and the $k_{\text {prop }}$ position of the lines at different excitation wavelengths, the dispersion curves of the different wire and slot waveguides are entirely determined as shown in Fig. 3 [10]. The narrowing of the waveguide width and the presence of the slot are accompanied with a decrease of the modal effective index as a result of the guided mode spreading into the surrounding low-index material. For the slot waveguides with given dimensions only the TM modes are guided. The comparison of these experimental dispersion curves with simulations (continuous lines with empty circles) calculated by the film mode matching method [13] reveals a very good agreement for the $400 \mathrm{~nm}$ wide rectangular waveguide. Small discrepancies that are observed for other waveguides investigated can be assigned to larger uncertainties in their cross-sectional dimensions introduced upon fabrication. The deviation is most pronounced for the $\mathrm{S} 70$ waveguide $(70 \mathrm{~nm}$ wide slot), where the relative error in the slot thickness is expected to be the largest. Another effect that may play a role but was disregarded in the dispersion calculations is the influence of the mode leakage into the Si substrate, which is separated by only $1 \mu \mathrm{m}$ thick $\mathrm{SiO}_{2}$ layer from the waveguide core region.

The obtained dispersion curves are strictly identical when using the $\mathrm{S}$ or $\mathrm{D}$ lines, which a posteriori confirms the $1.28 \mu \mathrm{m}$ periodic corrugations at the sidewalls. In addition, the results for $Y=1 \mu \mathrm{m}$ and $Y=3 \mu \mathrm{m}$ are strictly identical in the resolution limit
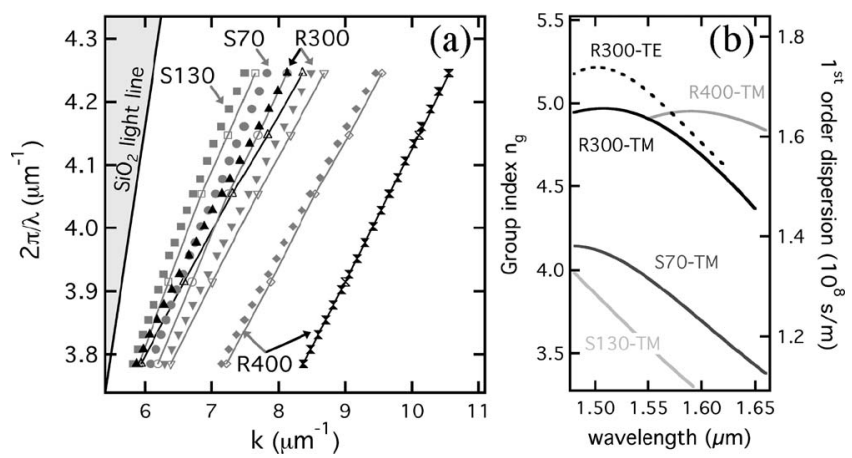

Fig. 3. (a) Experimental (dots) and theoretical (dots and line) dispersion curves of the TE (black) and TM (gray) modes propagating in the rectangular and slot nanowires. (b) Experimental group index dispersion curves. of the setup, which demonstrates the noninvasive nature of the LPGs approach. Note also that the determination of the wave vector of the TE modes of the $400 \mathrm{~nm}$ wide waveguide corresponds to an effective NA of the far-field microscope as high as 2.5.

In Fig. 3(b) the first-order dispersion coefficients are deduced from the experimental dispersion data obtained in Fig. 3(a). The observed anomalous dispersion results from the effect of the subwavelength waveguide geometry that dominates the material dispersion $[6,8]$. These data confirm that not only properly designed rectangular wire waveguides [3] but also slot waveguides allow wavelength separated laser beams to be phase matched at $\sim 1.55 \mu \mathrm{m}$ for nonlinear interactions.

To conclude, a deep investigation of intentionally induced and intrinsic optical losses in the far field allows us to retrieve the dispersive properties of the $\mathrm{Si}$ nanowire and slot waveguides, which are in excellent agreement with simulation results. Beyond, residual scattering losses resulting from the waveguide fabrication process carry a sufficient amount of information to noninvasively determine the sidewall roughness of photonic waveguide structures. Hence, the introduced Fourier-imaging technique is a powerful tool for an experimental control of both dispersion properties, e.g., with respect to optimized nonlinear device operations, and of fabrication tolerances of $\mathrm{Si}$ nanophotonic waveguide devices.

The authors acknowledge support from the European projects ePIXnet (IST-004525), Funfox (IST004582), the COST P11 action, and the Swiss NCCRQuantum Photonics.

\section{References}

1. Y. A. Vlasov and S. J. McNab, Opt. Express 12, 1622 (2004).

2. E. Bradley and D. G. Hall, Opt. Lett. 7, 235 (1982).

3. R. Dekker, A. Driessen, T. Wahlbrink, C. Moormann, J. Niehusmann, and M. Först, Opt. Express 14, 8336 (2006).

4. M. A. Foster, A. C. Turner, J. E. Sharping, B. S. Schmidt, M. Lipson, and A. L. Gaeta, Nature 441, 960 (2006).

5. V. R. Almeida, Q. Xu, C. A. Barrios, and M. Lipson, Opt. Lett. 29, 1209 (2004).

6. E. Dulkeith, F. Xia, L. Schares, W. M. J. Green, and Y. A. Vlasov, Opt. Express 14, 3853 (2006).

7. Q. Xu, V. R. Almeida, R. R. Panepucci, and M. Lipson, Opt. Lett. 29, 1626 (2004).

8. A. C. Turner, C. Manolatou, B. S. Schmidt, M. Lipson, M. A. Foster, J. E. Sharping, and A. L. Gaeta, Opt. Express 14, 4357 (2006).

9. H. K. Tsang, C. S. Wong, T. K. Liang, I. E. Day, S. W. Roberts, A. Harpin, J. Drake, and M. Asghari, Appl. Phys. Lett. 80, 416 (2002).

10. N. Le Thomas, R. Houdré, L. H. Frandsen, J. FagePedersen, A. V. Lavrinenko, and P. I. Borel, Phys. Rev. B 76, 035103 (2007).

11. J. W. Goodman, Introduction to Fourier Optics, 3rd ed. (Roberts \& Company, 2005).

12. C. A. Flory, IEEE J. Quantum Electron. 40, 949 (2004).

13. A. S. Sudbo, Pure Appl. Opt. 3, 381 (1994). 